DOI: $10.6060 / \mathrm{mhc} 2012.120468 \mathrm{p}$

\title{
Facile Synthesis of $15^{2}-$ Carboxamides of Methyl Pheophorbide $a$
}

\author{
Nikita O. Dugin, ${ }^{a}$ Maria G. Zavialova ${ }^{a}{ }^{a}$ Roman A. Novikov, ${ }^{\mathrm{b}}{ }$ Vladimir P. Timofeev, \\ Alexander Yu. Misharin, ${ }^{a}$ and Gelii V. Ponomareva@ \\ a Orekhovich Institute of Biomedical Chemistry RAMS, 119121 Moscow, Russia \\ ${ }^{\mathrm{b}}$ Engelhardt Institute of Molecular Biology RAS, 119991 Moscow, Russia \\ @Corresponding authorE-mail: gelii@yandex.ru
}

\begin{abstract}
The novel synthesis of chlorin $15^{2}$-carboxamides from methyl pheophorbide, which passed without cleavage of exocycle $E$, is developed. The combination of this approach together with known method of opening exocycle E by amines allows easy prepartion of $13^{l}$ - and $15^{2}$-carboxamides of chlorin $e_{6}$ and methyl pheophorbide a, comprising various substituents in amide moieties. Two examples (where substituents are either benzyl-, or hexadecyl-) are presented.
\end{abstract}

Keywords: Chlorin $e_{6}$, methyl pheophorbide $a$, amidation

Tetrapyrrol macrocycles of chlorin family are widely used as optical and fluorescent sensors in various biological studies, as well as sensitizers for tumor photodynamic therapy. ${ }^{[1-7]}$ Chemical modification of periphery substituents allows significantly change physical, chemical, spectral and photochemical properties of macrocycle and improve its solubility in various media, affinity to specific targets and photodynamic applications. A large number of chlorin derivatives comprising macrocycle modified with polyamines, amino acids, peptides, ${ }^{[8-14]}$ carbohydrates, ${ }^{[15]}$ steroids and lipids ${ }^{[16-18]}$ have been synthesized and used. Therefore development of new convenient methods for introducing of substituents in various positions of chlorin macrocycle is of importance.

Herein we present a simple method for synthesis of chlorin $15^{2}$-carboxamides from available methyl pheophorbide $a,{ }^{\#}$ which passed without cleavage of exocycle
$E$. The combination of this approach with the known method of nucleophilic opening of exocycle $E^{[19]}$ by amines allows easily prepare regioisomeric $13^{1}$ - and $15^{2}$-carboxamides of chlorin $e_{6}$ comprising various substituents in amide moieties (Scheme 1).

We discovered that interaction of methyl pheophorbide a 1 with 5 equivalents of amine (benzyl amine and hexadecyl amine were used) in boiling dioxane for $8 \mathrm{~h}$ led to $15^{2}$-carboxamides $\mathbf{2}$ and $\mathbf{3}$, respectively." The presence of exocyclic ring in compounds $\mathbf{2}$ and $\mathbf{3}$ was unequivocally demonstrated by absorption spectra (Figure 1), as well as by ${ }^{13} \mathrm{C}$ NMR spectra, displayed resonances characteristic for carbonyl groups (192.06 ppm and $192.50 \mathrm{ppm}$ for compounds $\mathbf{2}$ and $\mathbf{3}$, respectively). The yield of target products $\mathbf{2}$ and $\mathbf{3}$ in this reaction exceeds $80 \%$; besides we have found chlorin derivatives comprising opened exocyclic ring (8-11\%). These by-products were identified as $13^{1}$-carboxamides

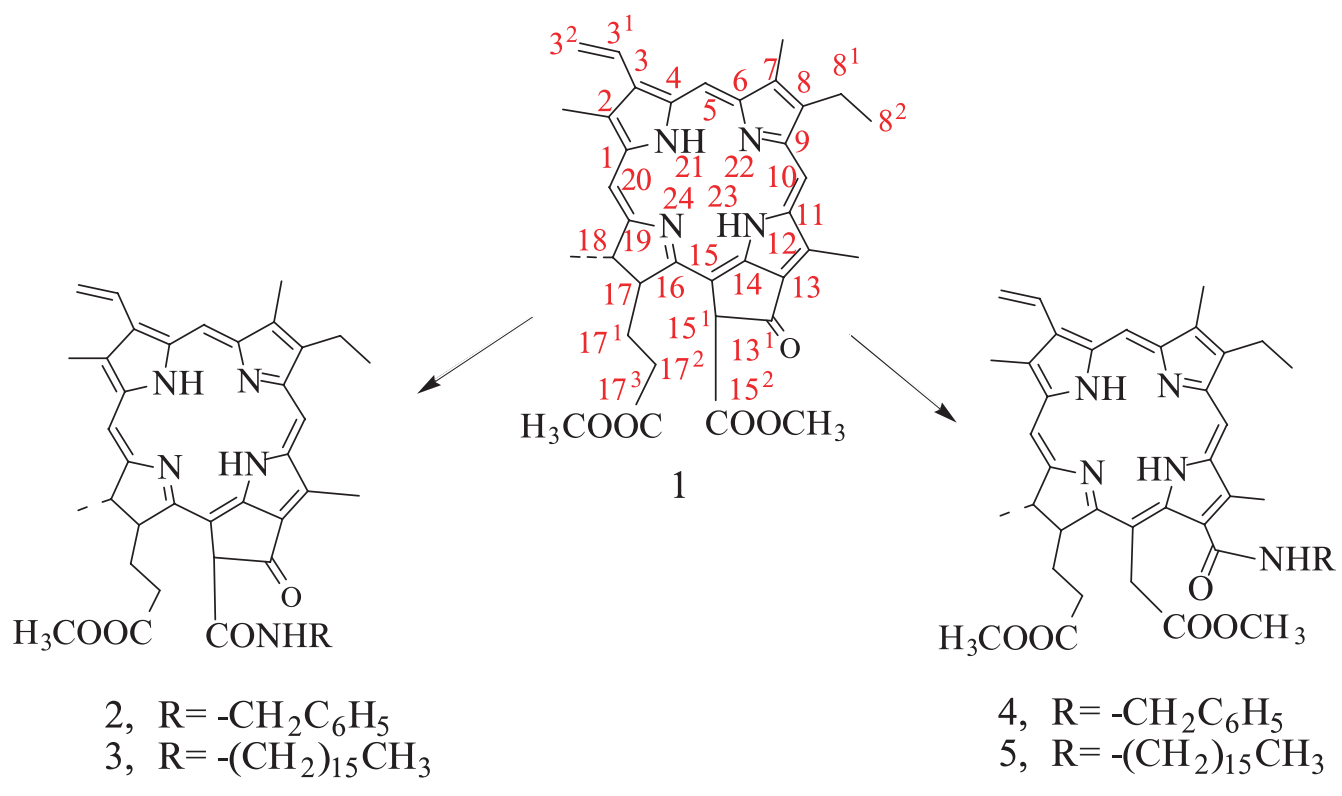

Scheme 1. 


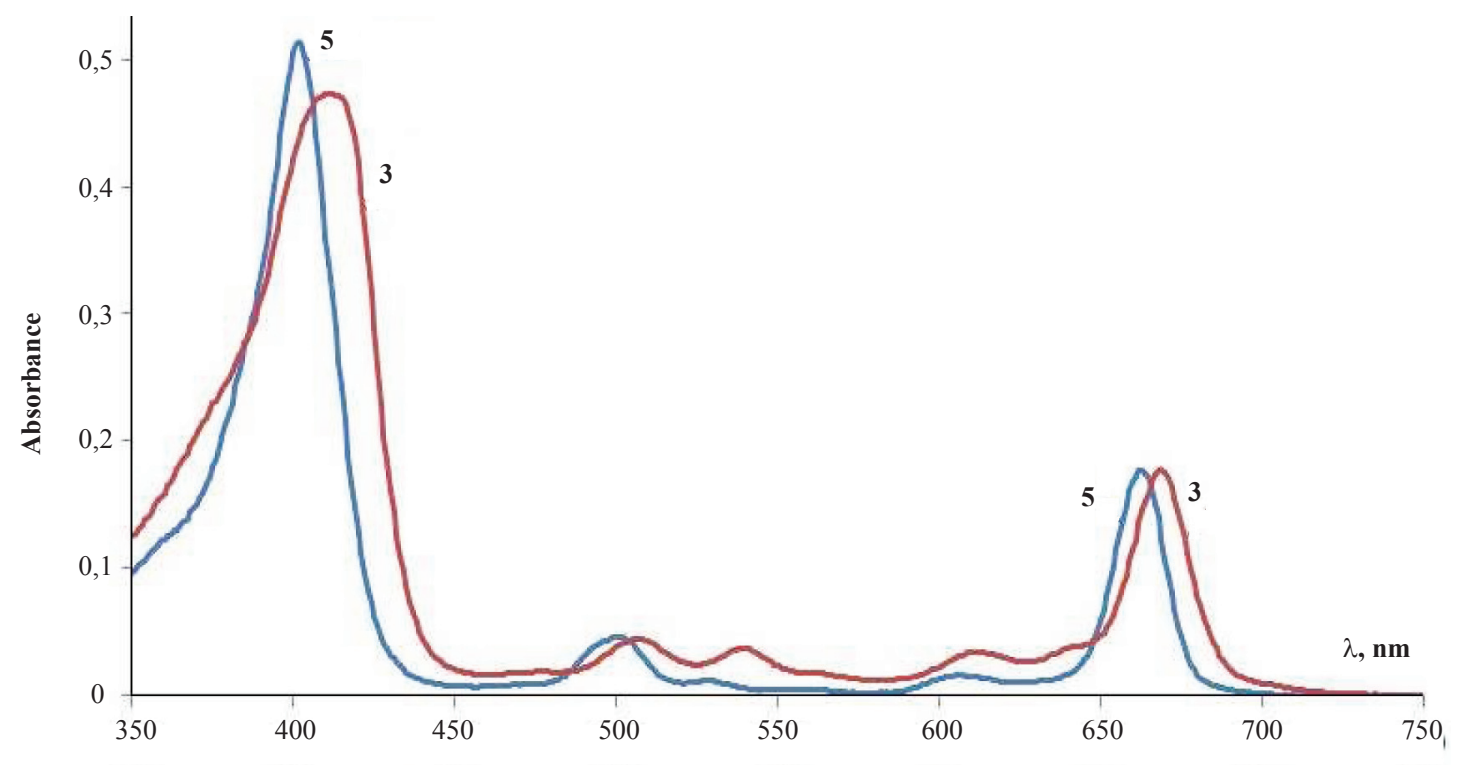

Figure 1. Absorption spectra of compounds $\mathbf{3}$ and $\mathbf{5}$ in $\mathrm{CH}_{2} \mathrm{Cl}_{2}$ (spectra of compounds $\mathbf{2}$ and $\mathbf{4}$ were identical to those for related counterparts).

4 and $5^{\wedge}$ (identification was carried out by comparison of their HRMS, ${ }^{1} \mathrm{H}$ NMR and absorption spectra with those for authentic samples $\mathbf{4}$ and $\mathbf{5}$ prepared according to reported methods ${ }^{[20-22]}$ ).

We concluded that nucleophilic substitution of $15^{2}$ methyl ester for amine and nucleophilic opening of exocyclic ring by amine in methyl pheophorbide $a \mathbf{1}$ are independent. The use of non volatile amines such as benzyl amine and hexadecyl amine, high temperature of reaction, and effect of $\beta$-carbonyl group in methyl pheophorbide $a \mathbf{1}$ promote formation of $15^{2}$ - carboxamide, which proceeds without exocyclic ring cleavage. On the other hand, prolong reaction at a relatively low temperature (THF, $35-40^{\circ} \mathrm{C}, 14-72 \mathrm{~h}$, depending on the structure of amine used) is in favor to nucleophilic opening of exocyclic ring.

Exocyclic ring in $15^{2}$-carboxamides $\mathbf{2}$ and $\mathbf{3}$ was more resistant to nucleophilic opening by amines in comparison with that in methyl pheophorbide $a \mathbf{1}$. Independent experiment showed that incubation of $15^{2}$-carboxamide 3 with benzyl amine in THF at $40^{\circ} \mathrm{C}$ for $72 \mathrm{~h}$ resulted in only traces of related $13^{1}, 15^{2}$-dicarboxamide $(<3 \%)$. This difference in reactivity of exocycle ring $E$ in compounds $\mathbf{1}$ and $\mathbf{3}$ towards amines was apparently due to different electron withdrawing effects of either ester, or amide groups.

All indicated compounds $\mathbf{2 - 5}$ were isolated in pure forms, their structures were fully confirmed by HRMS, ${ }^{1} \mathrm{H}$ NMR, ${ }^{13} \mathrm{C}$ NMR and absorption spectra. Taken together, data presented above revealed that reaction of methyl pheophorbide a $\mathbf{1}$ with amines allows simple preparation of either $13^{1}$-caboxamides with opened exocycle $E$, or $15^{2}$ carboxamides with saved exocycle $E$, depending on reaction conditions. The choice of conditions is very important: recent publications ${ }^{[22,23]}$ revealed that reaction of methyl pheophorbide $a \mathbf{1}$ with secondary amines in boiling toluene for $1.5-2 \mathrm{~h}$ led to mixtures of related $13^{1}$-carboxamides with opened exocylce $E$ and $15^{2}$-carboxamides with saved exocycle $E$.
Acknowledgements. This study was supported by Russian Foundation for Basic Research (grants RFBR 11-04-01940-a, RFBR 11-04-01537-a) and Program "Molecular and cell biology" of Presidium of Russian Academy of Sciences.

\section{Notes and References}

\# Absorption spectra were registered with a 'Thermospectronic Helios $\alpha$ ' spectrophotometer in $\mathrm{CH}_{2} \mathrm{Cl}_{2} ;{ }^{1} \mathrm{H}$ NMR and ${ }^{13} \mathrm{C} \mathrm{NMR}$ spectra - with an 'AMX-III' $400 \mathrm{MHz}$ Bruker instrument in $\mathrm{CDCl}_{3}$; high resolution mass spectra (HMRS) - with a Bruker 'Apex Ultra' FT ICR MS instrument at ion positive electro spray ionization mode. Flash chromatography was performed on silica gel G (0.015- 0.040 mm), analytical TLC - on UV254HPTLC silica gel plates, preparative TLC - on UV254-PTLC silica gel plates "Merck"; methyl pheophorbide $a \mathbf{1}$ was isolated from Spirulina platencis.

- Methyl 152-(benzylcarbamoyl)pheophorbide a, 2. HRMS, calculated for $\left[\mathrm{C}_{42} \mathrm{H}_{44} \mathrm{~N}_{5} \mathrm{O}_{4}\right]^{+}: 682.3393$, found: $682.3378 .{ }^{1} \mathrm{H}$ NMR $\delta_{\mathrm{H}}$ ppm: $8.56,9.38,9.51$ (each $\left.1 \mathrm{H}, \mathrm{s}, 5-, 10-, 20-\mathrm{H}\right)$, $7.99\left(1 \mathrm{H}, \mathrm{dd}, J=11.6 \mathrm{~Hz}\right.$ and $\left.J=17.8 \mathrm{~Hz}, 3^{1}-\mathrm{H}\right), 7.42(2 \mathrm{H}, \mathrm{d}$, $J=7.4 \mathrm{~Hz}, o-\mathrm{Ph}), 7.33(2 \mathrm{H}, \mathrm{t}, J=7.4 \mathrm{~Hz}, m-\mathrm{Ph}), 7.26$ (1H. t, $J=7.4 \mathrm{~Hz}, p-\mathrm{Ph}), 6.28(1 \mathrm{H}, \mathrm{dd}, J=17.8 \mathrm{~Hz}$ and $J=1.5 \mathrm{~Hz}$, $\left.3^{2}-\mathrm{H}, c i s\right), 6.17\left(1 \mathrm{H}, \mathrm{dd}, J=11.6 \mathrm{~Hz}, J=1.5 \mathrm{~Hz}, 3^{2}-\mathrm{H}\right.$, trans $)$, $4.67\left(2 \mathrm{H}, \mathrm{m}, \mathrm{CH}_{2} \mathrm{Ph}\right), 4.45(2 \mathrm{H}, \mathrm{dt}, J=8.7 \mathrm{~Hz}$ and $J=2.0 \mathrm{~Hz}$, $\left.17^{1}-\mathrm{H}\right), 4.38\left(1 \mathrm{H}, \mathrm{qd}, J=7.2 \mathrm{~Hz}, J=2.0 \mathrm{~Hz}, 8^{1}-\mathrm{H}\right), 3.23,3.40$, $3.55,3.68$ (each $3 \mathrm{H}, \mathrm{s}, 2-, 7-, 12-$ and $\left.\mathrm{O}-\mathrm{CH}_{3}\right), 2.20-2.34(2 \mathrm{H}$, $\left.\mathrm{m}, 17^{2}-\mathrm{H}\right), 1.81\left(3 \mathrm{H}, \mathrm{d}, J=7.3 \mathrm{~Hz}, 18-\mathrm{CH}_{3}\right) ; 1.70(3 \mathrm{H}, \mathrm{t}, J=$ $\left.7.2 \mathrm{~Hz}, 8^{2}-\mathrm{CH}_{3}\right),-1.60(1 \mathrm{H}, \mathrm{s}, \mathrm{NH}) .{ }^{13} \mathrm{C}$ NMR $\delta_{\mathrm{C}} \mathrm{ppm}: 11.25$; $12.09 ; 12.14 ; 17.40 ; 19.48 ; 23.07 ; 29.97 ; 30.92 ; 44.19 ; 50.25$; $51.20 ; 51.68 ; 65.44 ; 93.24 ; 97.47 ; 104.29 ; 105.31 ; 122.71$; $127.41 ; 127.96 ; 128.58 ; 128.69 ; 128.92 ; 129.16 ; 136.13$; $136.27 ; 136.55 ; 137.95 ; 138.47 ; 138.75 ; 141.98 ; 145.19$; $149.93 ; 150.95 ; 155.64 ; 162.73 ; 167.52 ; 172.26 ; 173.74$; 192.06. UV-vis $\lambda_{\max } \mathrm{nm}(\varepsilon): 411$ (96300); 504 (8200); 535 (7000); 604 (5200); 668 (35300).

Methyl 152-(hexadecylcarbamoyl)pheophorbide a, 3. HRMS, calculated for $\left[\mathrm{C}_{51} \mathrm{H}_{70} \mathrm{~N}_{5} \mathrm{O}_{4}\right]^{+}: 816.5428$, found: 816.5445. ${ }^{1} \mathrm{H}$ NMR $\delta_{\mathrm{H}}$ ppm: 8.56, 9.38, 9.50 (each 1H, s, 5-, 10-, 20-H), 7.99 $\left(1 \mathrm{H}, \mathrm{dd}, J=11.6 \mathrm{~Hz}\right.$ and $\left.J=17.8 \mathrm{~Hz}, 3^{1}-\mathrm{H}\right) ; 6.95(1 \mathrm{H}, \mathrm{t}, J=$ 
$7.0 \mathrm{~Hz}, \mathrm{NH}) ; 6.28\left(1 \mathrm{H}, \mathrm{dd}, J=17.8 \mathrm{~Hz}\right.$ and $J=1.5 \mathrm{~Hz}, 3^{2}-\mathrm{H}$, cis); $6.16\left(1 \mathrm{H}, \mathrm{dd}, J=11.6 \mathrm{~Hz}, J=1.5 \mathrm{~Hz}, 3^{2}-\mathrm{H}\right.$, trans $) ; 4.55$ $\left(2 \mathrm{H}, \mathrm{dt}, J=8.7 \mathrm{~Hz}\right.$ and $\left.J=2.0 \mathrm{~Hz}, 17^{1}-\mathrm{H}\right) ; 4.40(1 \mathrm{H}, \mathrm{qd}, J=7.2$ $\left.\mathrm{Hz}, J=2.0 \mathrm{~Hz}, 8^{1}-\mathrm{H}\right) ; 3.23,3.39,3.56,3.67$ (each 3H, s, 2-, 7-, 12- and $\left.\mathrm{O}_{-} \mathrm{CH}_{3}\right) ; 2.21-2.34\left(2 \mathrm{H}, \mathrm{m}, 17^{2}-\mathrm{H}\right) ; 1.88(3 \mathrm{H}, \mathrm{d}, J=7.3$ $\left.\mathrm{Hz}, 18-\mathrm{CH}_{3}\right) ; 1.70\left(3 \mathrm{H}, \mathrm{t}, J=7.2 \mathrm{~Hz}, 8^{2}-\mathrm{CH}_{3}\right) ; 1.25$ (br., $\mathrm{CH}_{2}$ in hexadecyl moiety); $0.86\left(3 \mathrm{H}\right.$, as. t, $\mathrm{CH}_{3}$ in hexadecyl moiety); -1.60 (br. s, NH). ${ }^{13} \mathrm{C}$ NMR $\delta_{\mathrm{C}}$ ppm: $11.32 ; 12.16 ; 14.17 ; 17.47$; $19.58 ; 22.77 ; 23.21 ; 23.80 ; 29.44 ; 29.64 ; 29.69 ; 29.74 ; 29.77$; $29.85 ; 30.12 ; 31.05 ; 32.01 ; 37.14 ; 38.70 ; 40.38 ; 50.33 ; 51.32$; $51.71 ; 53.48 ; 65.41 ; 67.92 ; 93.33 ; 97.52,104.32 ; 105.56$; $122.76 ; 128.70 ; 128.94 ; 129.28 ; 130.95 ; 131.92 ; 136.18$; $136.22 ; 136.34 ; 136.66 ; 138.03 ; 142.05 ; 145.26 ; 149.98$; $151.02 ; 155.72 ; 163.08 ; 167.35 ; 172.37 ; 173.80 ; 192.50$. UVvis $\lambda_{\text {max }} \mathrm{nm}(\varepsilon)$ : 411 (96300); 506 (8400); 539 (7300); 611 (5700); 669 (35900).

- $13^{I}$-(Benzylcarbamoyl)chlorin, 4. HRMS, calculated for $\left[\mathrm{C}_{43} \mathrm{H}_{48} \mathrm{~N}_{5} \mathrm{O}_{5}\right]^{+}$: 714.8845; found: 714.8852. ${ }^{1} \mathrm{H}$ NMR $\delta_{\mathrm{H}} \mathrm{ppm}$ : $8.80,9.61,9.86$ (each $1 \mathrm{H}, \mathrm{s}, 5-, 10-, 20-\mathrm{H}) ; 8.05(1 \mathrm{H}, \mathrm{dd}, J$ $=11.6 \mathrm{~Hz}$ and $\left.J=17.8 \mathrm{~Hz}, 3^{1}-\mathrm{H}\right) ; 7.55(2 \mathrm{H}, \mathrm{d} . J=7.4 \mathrm{~Hz}$, $o-\mathrm{Ph}) ; 7.42(2 \mathrm{H}, \mathrm{t}, J=7.4 \mathrm{~Hz}, m-\mathrm{Ph}) ; 7.34$ (1H. t, $J=7.4$ $\mathrm{Hz}, p-\mathrm{Ph}) ; 6.81(1 \mathrm{H}, \mathrm{t}, J=7.0 \mathrm{~Hz}, \mathrm{NH}) ; 6.33(1 \mathrm{H}, \mathrm{dd}, J=$ $17.8 \mathrm{~Hz}$ and $\left.J=1.5 \mathrm{~Hz}, 3^{2}-\mathrm{H}, c i s\right) ; 6.12(1 \mathrm{H}, \mathrm{dd}, J=11.6$ $\mathrm{Hz}, J=1.5 \mathrm{~Hz}, 3^{2}-\mathrm{H}$, trans); 5.28 and 5.55 (each $1 \mathrm{H}, \mathrm{d}, J=$ $\left.18.9 \mathrm{~Hz}, 15^{1}-\mathrm{H}\right) ; 4.78(1 \mathrm{H}, \mathrm{dd}, J=5.0 \mathrm{~Hz}$ and $J=14.5 \mathrm{~Hz}$, $\left.17^{2}-\mathrm{H}\right) ; 5.06\left(1 \mathrm{H}, \mathrm{dd}, J=6.1 \mathrm{~Hz}\right.$ and $\left.J=14.5 \mathrm{~Hz}, 17^{2}-\mathrm{H}\right)$; $4.47\left(2 \mathrm{H}, \mathrm{m}, \mathrm{CH}_{2} \mathrm{Ph}\right) ; 4.39(1 \mathrm{H}, \mathrm{qd}, J=7.2 \mathrm{~Hz}, J=2.0 \mathrm{~Hz}$, $\left.8^{1}-\mathrm{H}\right) ; 3.29,3.48,3.53,3.62,3.72$ (each $3 \mathrm{H}, \mathrm{s}, 2-, 7-, 12-$ and $\left.\mathrm{O}-\mathrm{CH}_{3}\right) ; 2.22-2.35\left(2 \mathrm{H}, \mathrm{m}, 17^{2}-\mathrm{H}\right) ; 1.72(3 \mathrm{H}, \mathrm{d}, J=7.3$ $\left.\mathrm{Hz}, 18-\mathrm{CH}_{3}\right) ; 1.71\left(3 \mathrm{H}, \mathrm{t}, J=7.2 \mathrm{~Hz}, 8^{2}-\mathrm{CH}_{3}\right) ;-1.77$ (s, NH). ${ }^{13} \mathrm{C} \mathrm{NMR} \delta_{\mathrm{C}}$ ppm: $11.35 ; 12.03 ; 12.18 ; 17.72 ; 19.72 ; 23.09$; $23.91 ; 29.15 ; 29.82 ; 31.24 ; 38.03 ; 44.93 ; 49.34 ; 51.63 ; 52.19$; $53.19 ; 67.66 ; 93.71 ; 98.90 ; 101.46 ; 107.94 ; 121.64 ; 127.84$; 128,$36 ; 128.96 ; 129.53 ; 129.96 ; 130.24 ; 134.64 ; 134.93$; $135.03 ; 135.08 ; 136.13 ; 137.99 ; 138.99 ; 144.82 ; 149.16$; $154.33 ; 166.72 ; 168.91 ; 169.37 ; 173.55 ; 174.02$. UV-vis $\lambda_{\max }$ nm (ع): 402 (104000); 500 (9100); 528 (2000); 607 (3000); $663(35800)$.

$13^{1}$-(Hexadecylcarbamoyl)chlorin, 5. HRMS, calculated for $\left[\mathrm{C}_{52} \mathrm{H}_{74} \mathrm{~N}_{5} \mathrm{O}_{5}\right]^{+}:$848.5690; found: 848.5673. ${ }^{1} \mathrm{H}$ NMR $\delta_{\mathrm{H}}$ ppm: $8.80,9.63,9.69$ (each 1H, s, 5-, 10-, 20-H); 8.08 (1H, dd, $J=$ $11.6 \mathrm{~Hz}$ and $\left.J=17.8 \mathrm{~Hz}, 3^{1}-\mathrm{H}\right) ; 6.40(1 \mathrm{H}, \mathrm{t}, J=7.0 \mathrm{~Hz}, \mathrm{NH})$; $6.35\left(1 \mathrm{H}, \mathrm{dd}, J=17.8 \mathrm{~Hz}\right.$ and $\left.J=1.5 \mathrm{~Hz}, 3^{2}-\mathrm{H}, c i s\right) ; 6.13(1 \mathrm{H}$, $\mathrm{dd}, J=11.6 \mathrm{~Hz}, J=1.5 \mathrm{~Hz}, 3^{2}-\mathrm{H}$, trans $), 4.55(\mathrm{dt}, J=8.7 \mathrm{~Hz}$ and $\left.J=2.0 \mathrm{~Hz}, 17^{1}-\mathrm{H}\right) ; 4.40(1 \mathrm{H}, \mathrm{qd}, J=7.2 \mathrm{~Hz}, J=2.0 \mathrm{~Hz}$, $1 \mathrm{H}, 8^{1}-\mathrm{H}$ ); 3.31, 3.48, 3.56, 3.60, 3.80 (each 3H, s, 2-, 7-, 12and $\left.\mathrm{O}-\mathrm{CH}_{3}\right) ; 2.21-2.34\left(2 \mathrm{H}, \mathrm{m}, 17^{2}-\mathrm{H}\right) ; 1.72(3 \mathrm{H}, \mathrm{d}, J=7.3$ $\left.\mathrm{Hz}, 18-\mathrm{CH}_{3}\right) ; 1.74\left(3 \mathrm{H}, \mathrm{t}, J=7.2 \mathrm{~Hz}, 8^{2}-\mathrm{CH}_{3}\right) ; 1.25$ (br., $\mathrm{CH}_{2}$ in hexadecyl moiety); 0.88 ( $3 \mathrm{H}$, as. t, $\mathrm{CH}_{3}$ in hexadecyl moiety); -1.79 (br. s, NH). ${ }^{13} \mathrm{C}$ NMR $\delta_{\mathrm{C}}$ ppm: $11.42 ; 12.08 ; 12.22 ; 14.19$; $17.68 ; 19.80 ; 22.78 ; 23.16 ; 23.90 ; 27.34 ; 29.21 ; 29.46 ; 29.61$; $29.69 ; 29.72 ; 29.76 ; 29.79,29.85 ; 30.12 ; 31.24 ; 32.02 ; 32.90$; $37.15 ; 37.99 ; 40.85 ; 48.14 ; 49.42 ; 50.10 ; 51.65 ; 52.20 ; 53.30$; $53.48 ; 63.12 ; 93.12 ; 94.14 ; 98.90 ; 101.38 ; 102.67 ; 121.94$; $128.84 ; 130.10 ; 134.93 ; 135.35 ; 135.93 ; 139.28 ; 144.53$; $149.20 ; 153.50 ; 167.32 ; 169.29 ; 173.56 ; 174.11$. UV-vis $\lambda_{\max }$ nm (ع): 402 (104000); 500 (9100); 528 (2000); 607 (3000); 663 (35800).

\section{References}

1. Dougherty T.J., Gomer C.J., Henderson B.W., Jori G., Kessel D., Korbelik M., Moan J., Peng Q. J. Natl. Cancer Inst. 1998, 90, 889-905.

2. Buytaert E., Dewaele M., Agostinis P. Biochim. Biophys. Acta 2007, 1776, 86-107.

3. Gariboldi M.B., Ravizza R., Baranyai P., Caruso E., Banfi S., Meschini S., Monti E. Bioorg. Med. Chem. 2009, 17, 20092016.

4. Dixon M.J., Bourrer L., MacRobert A.J., Eggleston I.M. Bioorg. Med. Chem. Lett. 2007, 17, 4518-4522.

5. Compagnin C., Moret F., Celotti L., Miotto G., Woodhams J.H., MacRobert A.J., Scheglmann D., Iratni S., Reddi E. J. Photochem. Photobiol. B 2008, 92, 91-97.

6. Borisov S.M., Papkovsky D.B., Ponomarev G.V., DeToma A.S., Safe R., Klimant I. J. Photochem. Photobiol. A 2009, 206, 87-92.

7. Girard D., Weagle G., Gupta A., Berruber G., Chapados C. Bioorg. Med. Chem. Lett. 2008, 18, 360-365.

8. Bisland S.K., Singh D, Gariepy J. Bioconjugate Chem. 1999, 10, 982-992.

9. Uzdensky A.B., Dergacheva O.Y., Zhavoronkova A.A., Reshetnikov A.V., Ponomarev G.V. Life Sci. 2004, 74, 21852197.

10. Zheng X., Morgan J., Pandey S., Chen Y., Tracy E., Baumann H., Missert J.R., Batt C., Jackson J., Bellnier D.A., Henderson B.W., Pandey R.K. J. Med. Chem. 2009, 52, 4306-4318.

11. Sibrian-Vazquez M., Jensen T.J., Fronczek F.R., Hammer R.P., Vicente M.G.H. Bioconjug. Chem. 2005, 16, 852-863.

12. Sibrian-Vazquez M., Jensen T.J., Vicente M.G.H. Org. Biomol. Chem. 2010, 8, 1160-1172.

13. Hargus J.A., Fronczek F.R., Vicente M.G.H., Smith K.M. Photochem. Photobiol. 2007, 83, 1006-1015.

14. Jensen T.J., Vicente M.G.H., Luguya R., Norton J., Fronczek F.R., Smith K.M. J. Photochem. Photobiol., B 2010, 100, 100111.

15. Jinadasa R.G.W., Hu X., Vicente M.G.H., Smith K.M. J. Med. Chem. 2011, 54, 7464-7476.

16. Dmitriev R.I., Ropiak H.M., Ponomarev G.V., Yashunsky D.V., Papkovsky D.B. Bioconjug. Chem. 2011, 22, 2507-2518.

17. Ol'shevskaya A.V., Nikitina R.G., Savchenko A.N., Malshakova M.V., Vinogradov A.M., Golovina G.V., Belykh D.V., Kutchin A.V., Kaplan M.A., Kalinin V.N., Kuzmin V.A., Shtil A.A. Bioorg. Med. Chem. 2009, 17, 1297-1306.

18. Nikolaeva I.A., Misharin A.Yu., Ponomarev G.V., Timofeev V.P., Tkachev Ya.V. Bioorg. Med. Chem. Lett. 2010, 20, 2872-2875.

19. Nikolaeva I.A., Morozova J.V., Zavialova M.G., Novikov R.A., Tkachev Ya.V., Timofeev V.P., Misharin A.Yu., Ponomarev G.V. Macroheterocycles 2010, 3, 150-156.

20. Ellsworth P.A., Storm C.B. J. Org. Chem. 1978, 43, 281-283.

21. Belykh D.V., Karmanova L.P., Spirikhin L.V., Kutchin A.V. Mendeleev Commun. 2002, 77.

22. Belykh D.V., Kopylov E.A., Gruzdev I.V., Kuchin A.V. Rus. J. Org. Chem. 2010, 46, 577-585.

23. Belykh D.V., Pushkareva E.I. Rus. J. Gen. Chem. 2011, 81, 1216-1281. 